\title{
Consideration of Instantaneous Space Vector for Permanent Magnet Synchronous Machine
}

\author{
Mineo TSUJI \\ Graduate School of Engineering \\ Nagasaki University \\ Bunkyo-machi 1-14 Nagasaki 852-8521, Japan \\ e-mail :mineo@nagasaki-u.ac.jp
}

\begin{abstract}
Physical meaning of the instantaneous space vector of salient permanent magnet synchronous machine is studied. For this purpose, we define a real physical space vectors. The electromagnetic torque is derived by current and flux densities. A space vector diagram in a transient state is derived and discussed.
\end{abstract}

Keywords- synchronous machine; instantaneous space vector; transient vector diagram; salient-pole machine; modeling

\section{INTRODUCTION}

In order to save energy, the applications of the interior permanent magnet synchronous machine (IPMSM) are expanding. Since the IPMSM does not have rotor winding, the copper loss is reduced than that of induction motor, and high power factor operation is possible. Furthermore, the application of IPMSM to the wind generation are studied actively.

Mathematical modeling and analysis of IPMSM using instantaneous space vector have been done in many literatures [1] [2] [4] [5]. However, the physical meaning of the instantaneous space vector of IPMSM is not completely considered including the transient operations. Especially the relation between the space vector of flux linkage and actual flux density is not clear in salient pole machine. Furthermore, the electromagnetic torque is derived by using the derivative of electromagnetic energy for salient pole machine in many literatures.

In order to solve these problems, we define the "Real Physical Space Vector" of current density, MMF, flux density and induced EMF. These vectors are useful to understand the conventional instantaneous space vectors physically.

\section{REAL PhysicAl SPACE Vector}

- Current, MMF and Flux Density-

Figure 1 shows a model of IPMSM and definition of positive direction of variables. $\boldsymbol{n}$ is a normal unit vector of plane of winding to compute a flux. Therefore $\boldsymbol{n}$ is positive direction of the flux. We assume that all windings are sinusoidally distributed windings as shown in Fig.2 [2][3]. When the phase currents flow, their current distributions per angle are defined as

$$
j_{a}(\theta)=\frac{N_{s} i_{a}}{2} \sin \theta
$$

$$
\begin{aligned}
& j_{b}(\theta)=\frac{N_{s} i_{b}}{2} \sin \left(\theta-\frac{2 \pi}{3}\right) \\
& j_{c}(\theta)=\frac{N_{s} i_{c}}{2} \sin \left(\theta+\frac{2 \pi}{3}\right)
\end{aligned}
$$

In order to consider the saliency, equivalent air-gap length is assumed as [3]

$$
g(\theta)=\frac{1}{\alpha_{1}-\alpha_{2} \cos 2\left(\theta-\theta_{r}\right)}
$$

where, $\alpha_{1}>0, \alpha_{2}>0$

The permeability of permanent magnet is considered to be equal to that of air. Therefore, the equivalent air-gap length is long in $d$-axis direction. Applying Ampere's law around the path of Fig. 3 and neglecting the field intensity of steel, the magnetic field intensity $H_{a}$ caused by $i_{a}$ in the air-gap is obtained as follows:

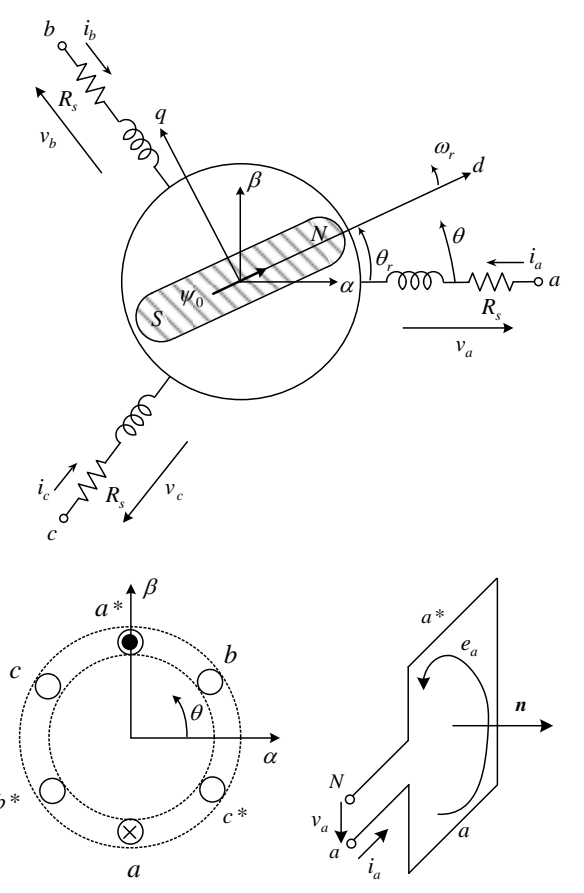

Figure 1. Model of IPMSM and definition of positive direction 


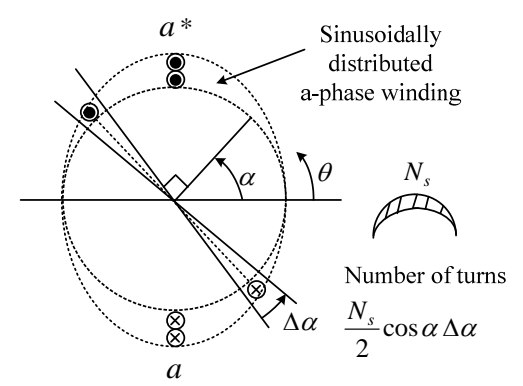

Figure 2. Sinusoidally distributed winding.

$$
H_{a}(\alpha)=\frac{1}{2 g(\alpha)} \int_{\alpha}^{\alpha+\pi} j_{a}(\theta) d \theta=\frac{N_{s} i_{a}}{2 g(\alpha)} \cos \alpha
$$

The positive direction of $\mathrm{H}$ is assumed from the rotor to the stator. The magnetomotive force (MMF) of $i_{a}$ which is used at point $\alpha$ is computed as

$$
F_{a}(\alpha)=\frac{1}{2} \int_{\alpha}^{\alpha+\pi} j_{a}(\theta) d \theta=\frac{N_{s} i_{a}}{2} \cos \alpha
$$

Similarly the MMFs of $i_{b}$ and $i_{c}$ are obtained respectively as

$$
\begin{aligned}
& F_{b}(\alpha)=\frac{N_{s} i_{b}}{2} \cos \left(\alpha-\frac{2}{3} \pi\right) \\
& F_{c}(\alpha)=\frac{N_{s} i_{c}}{2} \cos \left(\alpha+\frac{2}{3} \pi\right)
\end{aligned}
$$

The flux densities by phase currents are derived respectively as follows:

$$
\begin{aligned}
& B_{a}(\alpha)=\frac{\mu_{0}}{g(\alpha)} F_{a}(\alpha) \\
& B_{b}(\alpha)=\frac{\mu_{0}}{g(\alpha)} F_{b}(\alpha) \\
& B_{c}(\alpha)=\frac{\mu_{0}}{g(\alpha)} F_{c}(\alpha)
\end{aligned}
$$

By substituting (4) into (9)-(11), the following equations are obtained [3]

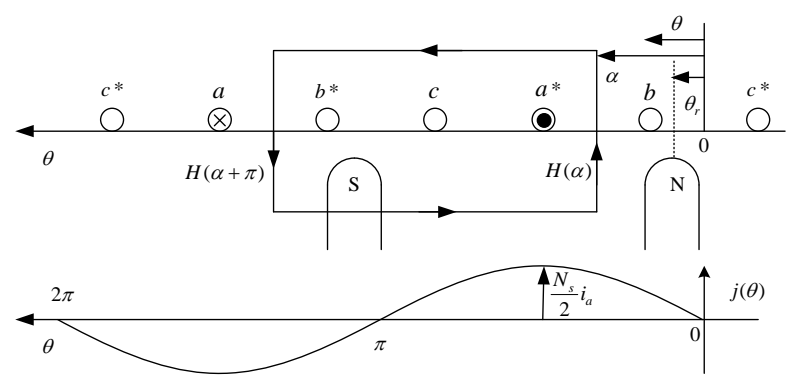

Figure 3. Current distribution.

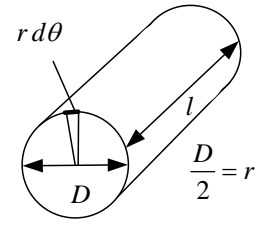

Figure 4. Dimensions.

$$
\begin{aligned}
& B_{a}(\alpha)=\frac{\mu_{0} N_{s} i_{a}}{2}\left(\alpha_{1}-\alpha_{2} \cos 2\left(\alpha-\theta_{r}\right)\right) \cos \alpha \\
& B_{b}(\alpha)=\frac{\mu_{0} N_{s} i_{b}}{2}\left(\alpha_{1}-\alpha_{2} \cos 2\left(\alpha-\theta_{r}\right)\right) \cos \left(\alpha-\frac{2 \pi}{3}\right) \\
& B_{c}(\alpha)=\frac{\mu_{0} N_{s} i_{c}}{2}\left(\alpha_{1}-\alpha_{2} \cos 2\left(\alpha-\theta_{r}\right)\right) \cos \left(\alpha+\frac{2 \pi}{3}\right)
\end{aligned}
$$

When $i_{a}+i_{b}+i_{c}=0$, the currents are expressed even in transient state as follows:

$$
\begin{aligned}
& i_{a}=I(t) \cos \theta_{i}(t) \\
& i_{b}=I(t) \cos \left(\theta_{i}(t)-\frac{2}{3} \pi\right) \\
& i_{c}=I(t) \cos \left(\theta_{i}(t)+\frac{2}{3} \pi\right)
\end{aligned}
$$

Our theory is developed by these expressions of (15) - (17).

The current density distribution caused by 3-phases currents is obtained by (1)-(3), (15)-(17) as

$$
\begin{aligned}
j_{a b c}(\alpha) & =j_{a}(\alpha)+j_{b}(\alpha)+j_{c}(\alpha) \\
& =\frac{3 N_{s}}{4} I(t) \sin \left(\alpha-\theta_{i}(t)\right)
\end{aligned}
$$

The MMF distribution caused by 3-phases currents is obtained by (6)-(8), (15)-(17) as

$$
\begin{aligned}
F_{a b c}(\alpha) & =F_{a}(\alpha)+F_{b}(\alpha)+F_{c}(\alpha) \\
& =\frac{3 N_{s}}{4} I(t) \cos \left(\alpha-\theta_{i}(t)\right)
\end{aligned}
$$

The air-gap flux density by phase currents and permanent magnet is derived by (12)-(14), (15)-(17) as

$$
\begin{aligned}
B_{a b c}(\alpha)=B_{a}(\alpha)+B_{b}(\alpha)+B_{c}(\alpha)+B_{0} \cos \left(\alpha-\theta_{r}\right) \\
=\frac{3 \mu_{0} N_{s} I(t)}{4}\left\{\alpha_{1} \cos \left(\theta_{i}-\alpha\right)-\frac{\alpha_{2}}{2}\left(\cos \left(\alpha-2 \theta_{r}+\theta_{i}\right)\right.\right. \\
\left.\left.\quad+\cos \left(3 \alpha-2 \theta_{r}-\theta_{i}\right)\right)\right\}+B_{0} \cos \left(\alpha-\theta_{r}\right)
\end{aligned}
$$

The distributions of current density, MMF and flux density can be observed in the motor. So we define "real physical space vector" of MMF (19) as

$$
\dot{F}_{a b c}(t)=\frac{3 N_{s}}{4} I(t) e^{j \theta_{i}(t)}
$$


The direction angle $\theta_{i}(t)$ of real physical space vector shows the angle a in which the MMF (19) becomes maximum. Similarly, the real physical space vector of current density (18) is expressed as

$$
\dot{j}_{a b c}=\frac{3 N_{s}}{4} j I(t) e^{j \theta_{i}(t)}=j \dot{F}_{a b c}
$$

In the case of flux density (20) , we can obtain the physical space vector by neglecting the third harmonic component as follows:

$$
\dot{B}_{a b c}=\frac{3 \mu_{0} N_{s} I(t)}{4}\left\{\alpha_{1} e^{j \theta_{i}}-\frac{\alpha_{2}}{2} e^{j\left(2 \theta_{r}-\theta_{i}\right)}\right\}+B_{0} e^{j \theta_{r}}
$$

\section{INSTANTANEOUS SPACE VECTOR}

-Flux Linkage and Electromagnetic Torque-

In general, the instantaneous space vector is defined as [1]

$$
\dot{f}_{s}(t)=\sqrt{\frac{2}{3}}\left(f_{a}(t)+e^{j \frac{2}{3} \pi} f_{b}(t)+e^{-j \frac{2}{3} \pi} f_{c}(t)\right)
$$

where, $f$ means phase voltage $v$, phase current $i$ and flux linkage $\psi$. It is well known that the space vector can be also expressed by using $\alpha-\beta$ stationary reference frame quantities.

$$
\dot{f}_{s}(t)=f_{\alpha}(t)+j f_{\beta}(t)
$$

Therefore,

$$
\dot{f}_{s}(t)=F(t) e^{j \theta_{f}(t)}
$$

where, $F(t)=\sqrt{f_{\alpha}^{2}(t)+f_{\beta}^{2}(t)}, \theta_{f}(t)=\tan ^{-1} \frac{f_{\beta}(t)}{f_{\alpha}(t)}$

By assuming $f_{a}+f_{b}+f_{c}=0$, we have

$$
\begin{aligned}
& f_{a}=\sqrt{2 / 3} F(t) \cos \theta_{f}(t) \\
& f_{b}=\sqrt{2 / 3} F(t) \cos \left(\theta_{f}(t)-2 \pi / 3\right) \\
& f_{c}=\sqrt{2 / 3} F(t) \cos \left(\theta_{f}(t)+2 \pi / 3\right)
\end{aligned}
$$

Equations of currents (15) - (17) are derived by (27) - (29) respectively. By using (15) - (17), the space vector of current is obtained as

$$
\dot{i}_{s}(t)=\sqrt{\frac{3}{2}} I(t) e^{j \theta_{i}(t)}
$$

From (22) and (30), we have

$$
\dot{F}_{a b c}(t)=\frac{3 N_{s}}{4} I(t) e^{j \theta_{i}(t)}=\frac{3 N_{s}}{4} \sqrt{\frac{2}{3}} \dot{i}_{s}(t)
$$

It means that the real physical space vector of MMF is proportional to the current space vector. It is noted that the actual distributions of current density is expressed by its real physical space vector (22). It is convenient that the relation can be explained by a right-handed screw rule. From (26), and (27)-(29), the rule is valid as shown in Fig.5. For example, when $\theta_{f}=0, f_{a}$ is maximum even in transient state. In order to satisfy the right-handed screw rule, the positive direction of current should be defined as shown Fig.1.
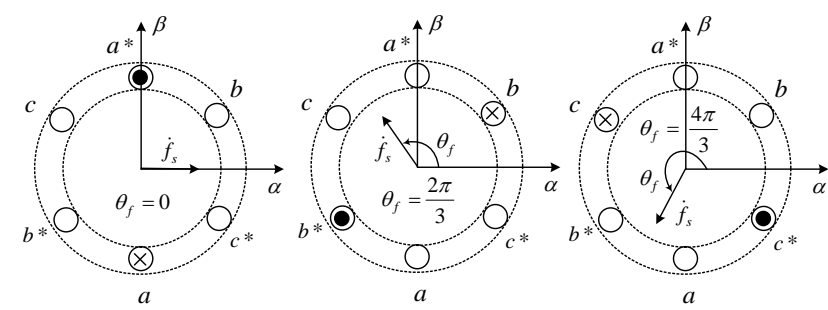

Figure 5. Physical meaning of instantaneous space vector.

The flux linkage of a-phase winding is obtained from (20) as

$$
\begin{aligned}
\psi_{a} & =\int_{-\frac{\pi}{2}}^{\frac{\pi}{2}} \frac{N_{s}}{2} \cos \alpha \int_{\alpha-\frac{\pi}{2}}^{\alpha+\frac{\pi}{2}} B_{a b c}(\theta) l r d \theta d \alpha+l_{s} i_{a} \\
& =L_{1} I(t) \cos \theta_{i}-L_{2} I(t) \cos \left(2 \theta_{r}-\theta_{i}\right)+\psi_{0} \cos \theta_{r}
\end{aligned}
$$

where,

$$
\begin{aligned}
& L_{1} \equiv \frac{3 \pi N_{s}^{2} \operatorname{lr} \mu_{0}}{8} \alpha_{1}+l_{s} \\
& L_{2} \equiv \frac{3 \pi N_{s}^{2} \operatorname{lr} \mu_{0} \alpha_{2}}{16} \\
& \psi_{0}=\frac{\pi N_{s} B_{0} \operatorname{lr}}{2} \\
& l_{s} \text { : leakage inductance }
\end{aligned}
$$

By (32), it is recognized that the third harmonics component $(3 \alpha)$ of the flux density (20) does not affect the instantaneous flux linkage because of sinusoidally distributed winding. The $b$ - and $c$-phases flux linkages are obtained similarly as

$$
\begin{aligned}
\psi_{b}= & L_{1} I(t) \cos \left(\theta_{i}-\frac{2 \pi}{3}\right)-L_{2} I(t) \cos \left(2 \theta_{r}-\frac{2 \pi}{3}-\theta_{i}\right) \\
& +\psi_{0} \cos \left(\theta_{r}-\frac{2 \pi}{3}\right) \\
\psi_{b}= & L_{1} I(t) \cos \left(\theta_{i}+\frac{2 \pi}{3}\right)-L_{2} I(t) \cos \left(2 \theta_{r}+\frac{2 \pi}{3}-\theta_{i}\right) \\
& +\psi_{0} \cos \left(\theta_{r}+\frac{2 \pi}{3}\right)
\end{aligned}
$$


The instantaneous space vector of the flux linkage can be obtained by (24), (32), (36) and (37) as follows:

$$
\dot{\psi}_{s}=\sqrt{\frac{3}{2}} L_{1} I(t) e^{j \theta_{i}}-\sqrt{\frac{3}{2}} L_{2} I(t) e^{j\left(2 \theta_{r}-\theta_{i}\right)}+\sqrt{\frac{3}{2}} \psi_{0} e^{j \theta_{r}}
$$

By using (30), the flux linkage is expressed as

$$
\begin{aligned}
& \dot{\psi}_{s}=L_{1} \dot{i}_{s}-L_{2} e^{j 2 \theta_{r}} \dot{i}_{s}^{*}+\psi e^{j \theta_{r}} \\
& \text { where, } \psi=\sqrt{\frac{3}{2}} \psi_{0}
\end{aligned}
$$

By comparing (23) and (38), we could prove theoretically that the instantaneous space vector of the flux linkage except leakage is proportional to the real physical space vector of flux density when the third harmonic component of flux density are neglected. That is,

$$
\dot{\psi}_{s}=\sqrt{\frac{3}{2}} \frac{\pi r l N_{s}}{2} \dot{B}_{a b c}
$$

The electromagnetic torque in the direction of rotation can be computed using Fleming's left-hand rule as

$$
\begin{aligned}
\tau_{e}= & -r \int_{0}^{2 \pi} j_{a b c}(\alpha) B_{a b c}(\alpha) l d \alpha \\
= & -r l \int_{0}^{2 \pi} \frac{3 N_{s}}{4} I(t) \sin \left(\alpha-\theta_{i}\right)\left[\frac { 3 \mu _ { 0 } N _ { s } I ( t ) } { 4 } \left\{\alpha_{1} \cos \left(\theta_{i}-\alpha\right)\right.\right. \\
& \left.-\frac{\alpha_{2}}{2}\left(\cos \left(\alpha-2 \theta_{r}+\theta_{i}\right)+\cos \left(3 \alpha-2 \theta_{r}-\theta_{i}\right)\right)\right\} \\
& \left.+B_{0} \cos \left(\alpha-\theta_{r}\right)\right] d \alpha \\
= & -\frac{3}{2} L_{2} I^{2}(t) 2 \sin \left(\theta_{i}-\theta_{r}\right) \cos \left(\theta_{i}-\theta_{r}\right) \\
& +\psi \sqrt{\frac{3}{2}} I(t) \sin \left(\theta_{i}-\theta_{r}\right) \\
= & \mathrm{I}_{\mathrm{m}}\left(-L_{2} e^{-j 2 \theta_{r}} \dot{i}_{s} \dot{i}_{s}+\psi e^{-j \theta_{r}} \dot{i}_{s}\right) \\
= & \mathrm{I}_{\mathrm{m}}\left(\dot{\psi}_{s}^{*} \dot{i}_{s}\right) \\
= & -\operatorname{Re}\left(\pi r l N_{s} \dot{B}_{a b c}^{*} \dot{j}_{a b c}\right)
\end{aligned}
$$

By (40), it is recognized that the third harmonics component $(3 \alpha)$ of the flux density (20) does not affect the instantaneous torque because of sinusoidally distributed winding. This relation was also obtained in the case of the flux linkage. Therefore, the third harmonics component will not affect the terminal voltages and phase currents. Therefore, it is reasonable to discuss the flux density by (23). In this case, $\dot{\psi}_{s}$ (except leakage inductance term) is proportional to flux density $\dot{B}_{a b c}$. So the instantaneous torque (41) is understood by Fleming's left-hand rule.

\section{Space Vector Diagram}

-Induced Electromotive Force and Terminal Voltage-

The voltage equation of IPMSM is expressed as

$$
\left[\begin{array}{c}
v_{a} \\
v_{b} \\
v_{c}
\end{array}\right]=R_{s}\left[\begin{array}{l}
i_{a} \\
i_{b} \\
i_{c}
\end{array}\right]+p\left[\begin{array}{l}
\psi_{a} \\
\psi_{b} \\
\psi_{c}
\end{array}\right]
$$

By using space vector, (42) becomes

$$
\begin{aligned}
\dot{v}_{s}= & \sqrt{\frac{2}{3}}\left\{R_{s} i_{a}+p \psi_{a}+e^{j \frac{2}{3}}\left(R_{s} i_{b}+p \psi_{b}\right)\right. \\
& \left.+e^{-j \frac{2}{3} \pi}\left(R_{s} i_{c}+p \psi_{c}\right)\right\} \\
= & R_{s} \dot{i}_{s}+p \dot{\psi}_{s}
\end{aligned}
$$

The space vector in a $d-q$ reference frame is defined as

$$
\dot{f}_{d q} \equiv e^{-j \theta_{r}} \dot{f}_{s}
$$

Therefore, the current space vector is expressed as

$$
\dot{i}_{s}=e^{j \theta_{r}} \dot{i}_{d q}
$$

By substituting (45) into (39), we have

$$
\begin{aligned}
\dot{\psi}_{s} & =\left(L_{1} \dot{i}_{d q}-L_{2} \dot{i}_{d q}^{*}+\psi\right) e^{j \theta_{r}} \\
& =\left(L_{d} \dot{i}_{d}+\psi+j L_{q} i_{q}\right) e^{j \theta_{r}}
\end{aligned}
$$

where, $L_{d}=L_{1}-L_{2}, L_{q}=L_{1}+L_{2}$

By substituting (46) into (43), we have

$$
\begin{aligned}
\dot{v}_{s}= & R_{s} \dot{i}_{s}+j \omega_{r} \psi e^{j \theta_{r}}+\left(-\omega_{r} L_{q} i_{q}+L_{d}\left(p i_{d}\right)\right) e^{j \theta_{r}} \\
& +j\left(\omega_{r} L_{d} i_{d}+L_{q}\left(p i_{q}\right)\right) e^{j \theta_{r}}
\end{aligned}
$$

The Park's equation in $d-q$ axis is obtained by using (44) in (47) and eliminating $e^{j \theta_{r}}$.

The induced electromotive force(EMF) by the permanent magnet ( the positive direction is defined in Fig.1) is obtained as

$$
\left[\begin{array}{l}
e_{a} \\
e_{b} \\
e_{c}
\end{array}\right]=-\frac{d}{d t} \psi_{0}\left[\begin{array}{c}
\cos \theta_{r} \\
\cos \left(\theta_{r}-\frac{2}{3} \pi\right) \\
\cos \left(\theta_{r}+\frac{2}{3} \pi\right)
\end{array}\right]
$$




$$
=\omega_{r} \psi_{0}\left[\begin{array}{c}
\sin \theta_{r} \\
\sin \left(\theta_{r}-\frac{2}{3} \pi\right) \\
\sin \left(\theta_{r}+\frac{2}{3} \pi\right)
\end{array}\right]
$$

The space vector of induced EMF is expressed as

$$
\dot{e}_{0}=\sqrt{\frac{2}{3}}\left(e_{a}+e_{b} e^{j \frac{2}{3} \pi}+e_{c} e^{-j \frac{2}{3} \pi}\right)=-j \omega_{r} \psi e^{j \theta_{r}}
$$

The counter EMF is obtained as

$$
\dot{e}_{0}{ }^{\prime}=-\dot{e}_{0}=j \omega_{r} \psi e^{j \theta_{r}}
$$

By substituting (50) into (47), we have

$$
\begin{aligned}
\dot{v}_{s}= & R_{s} \dot{i}_{s}+\dot{e}_{0}{ }^{\prime}+\left(-\omega_{r} L_{q} i_{q}+L_{d}\left(p i_{d}\right)\right) e^{j \theta_{r}} \\
& +j\left(\omega_{r} L_{d} \dot{i}_{d}+L_{q}\left(p i_{q}\right)\right) e^{j \theta_{r}}
\end{aligned}
$$

In section II we defined the real physical space vectors which are current density, MMF and flux density. In this section, we discuss the real physical space vector of the induced EMF (48). The currents are uniform in each phase. On the other hand, the induced EMF is different even in a same phase and it is a function of angle. Hence, we consider the winding as a conductor distributed in the space. The induced EMF density at the angle $\alpha$ can be expressed by Fleming's right-hand rule as

$$
e_{a b c}=N_{a b c} B_{0} l r \omega_{r} \cos \left(\alpha-\theta_{r}\right)
$$

where, $N_{\text {dis }}$ is the winding density considering all phases and can be expressed as

$$
\begin{aligned}
N_{a b c} & =\frac{N_{s}}{2}\left(|\sin \alpha|+\mid \sin \left(\alpha-\frac{2 \pi)}{3}|+| \sin \left(\alpha+\frac{2 \pi)}{3} \mid\right)\right.\right. \\
& \simeq N_{s}
\end{aligned}
$$

Using (52) and (53), the real physical space vector of induced EMF density is expressed as

$$
\dot{e}_{a b c} \simeq N_{s} B_{0} \operatorname{lr} \omega_{r} e^{j \theta_{r}}
$$

By using (51), we can draw a instantaneous space vector diagram shown in Figs.6 (motoring operation) and 7 (regenerating operation). In these figure, the space vector of the flux linkage (39) is also shown. The $e^{j 2 \theta_{r}} i_{s}^{*}$ is the mirrorphase current of $\dot{i}_{s}$ in regard to $d$-axis [6]. The winding denoted by the symbols $\otimes$ and $\odot$ shows the point of the real physical space vector. The $\dot{i}_{s}$ and $\dot{e}_{0}$ correspond to the $\dot{j}_{a b c}$ and $\dot{e}_{a b c}$ respectively.

In steady-state, $p=0$ in (51). Therefore,

$$
\dot{v}_{s}=R_{s} \dot{i}_{s}+j \omega_{r}\left(L_{d} i_{d}+j L_{q} i_{q}\right) e^{j \theta_{r}}+j \omega_{r} \psi e^{j \theta_{r}}
$$

Concerning the flux linkage, from (46)

$$
p \dot{\psi}_{s}=j \omega_{r}\left(L_{d} i_{d}+\psi+j L_{q} i_{q}\right) e^{j \theta_{r}}=j \omega_{r} \dot{\psi}_{s}
$$

Therefore, $p \dot{\psi}_{s}$ is orthogonal to the $\dot{\psi}_{s}$. By using (44), we have

$$
\dot{v}_{d q}=R_{s} \dot{i}_{d q}+j \omega_{r}\left(L_{d} i_{d}+j L_{q} i_{q}\right)+j \omega_{r} \psi
$$

where, $\dot{i}_{d q}=i_{d}+j i_{q}$

From (30), the conventional phasor of $a$-phase is obtained by dividing space vector $\dot{v}_{d q}, \dot{i}_{d q}$ by $\sqrt{3}$.

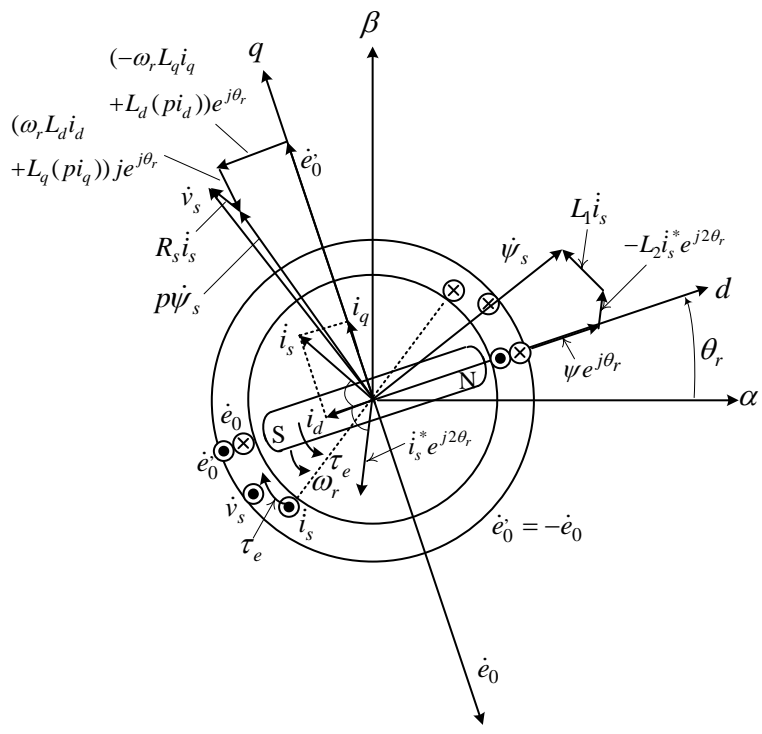

Figure 6. Transient space vector diagram of IPMSM (Motoring operation).

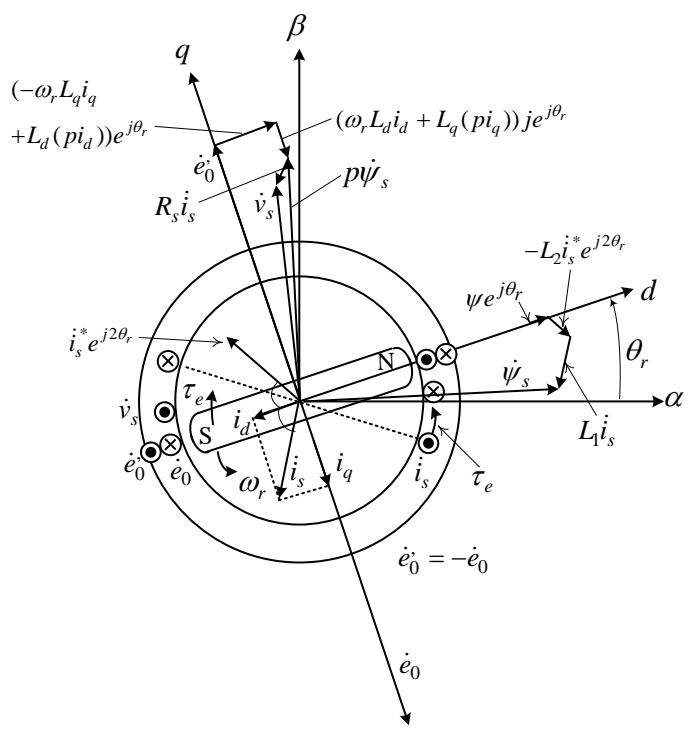

Figure 7. Transient space vector diagram of IPMSM (Regenarating operation). 


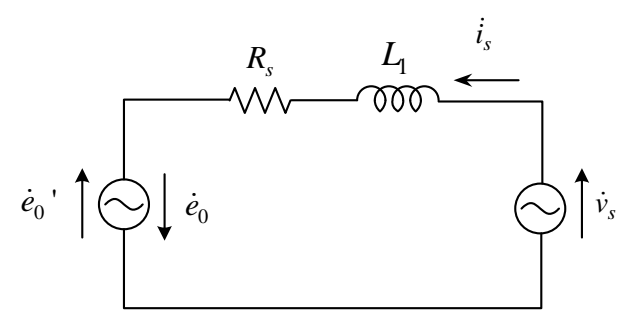

Figure 8. Transient equivalent circuit of non-salient PMSM.

For non-salient IPMSM, the following equation is valid.

$$
\alpha_{2}=0, L_{2}=0, L_{d}=L_{q}=L_{1}
$$

Eq. (51) becomes

$$
\begin{aligned}
\dot{v}_{S}= & R_{s} \dot{i}_{s}+\dot{e}_{0}{ }^{\prime}+L_{1}\left(-\omega_{r} i_{q}+p i_{d}\right) e^{j \theta_{r}} \\
& +j L_{1}\left(\omega_{r} i_{d}+p i_{q}\right) e^{j \theta_{r}} \\
= & R_{S} \dot{i}_{S}+p L_{1} \dot{i}_{S}+\dot{e}_{0}{ }^{\prime}
\end{aligned}
$$

By using (59), the equivalent circuit which is valid in transient state is shown in Fig.8 for non-salient motor. Figs.9 (motoring operation) and 10 (regenerating operation) show the instantaneous space vector diagrams. The steady state equation (57) becomes

$$
\dot{v}_{d q}=R_{s} \dot{i}_{d q}+j \omega_{r} L_{1} \dot{i}_{d q}+j \omega_{r} \psi
$$

\section{CONCLUSION}

The conclusions drawn from this paper are summarized as follows:

(1) We defined the "Real Physical Space Vector" of current density, MMF, flux density and induced EMF. These vectors are useful to understand the conventional instantaneous space vectors physically.

(2) We could prove theoretically that the instantaneous space vector of the flux linkage except leakage is proportional to the real physical space vector of flux density when the third harmonic component of flux density are neglected.

(3) The electromagnetic torque is derived by using the current density and the flux density distribution. By the definition of the real physical space vector, the torque equation is well understood.

(4) We showed the instantaneous space vector diagrams of IPMSM which are valid in transient state. In these figures, we can understand currents and induced EMF distribution also.

(5) Since the theory proposed in this paper is easy to understand physically, it is useful to the education of IPMSM.

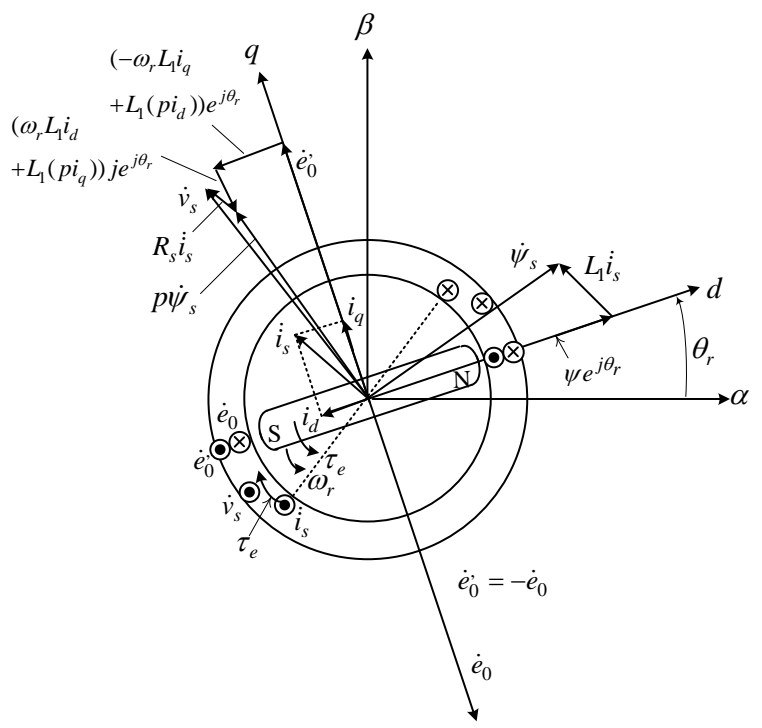

Figure 9. Transient space vector diagram of non-salient PMSM (Motoring operation).

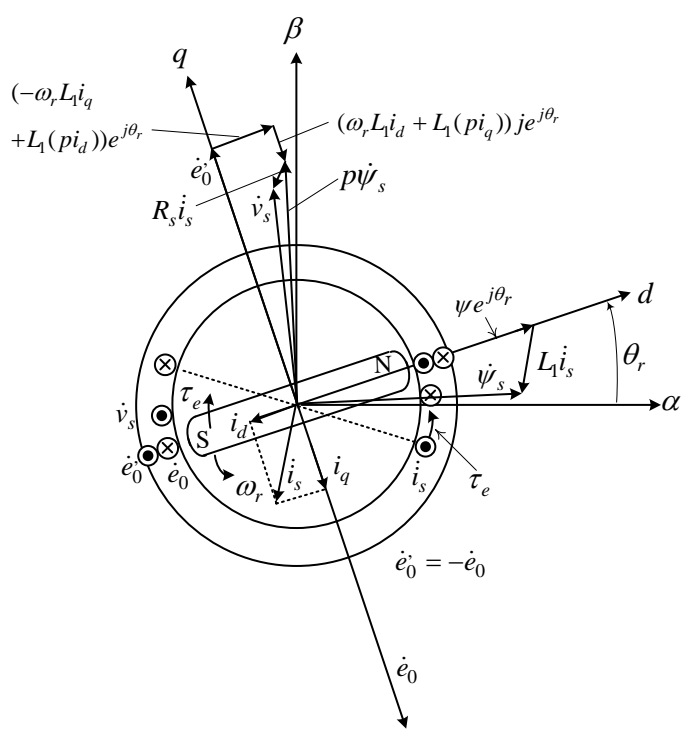

Figure 10. Transient space vector diagram of non-salient PMSM (Regenerating operation).

\section{REFERENCES}

[1] Pál K. Kovács : “Transient Phenomena in Electrical Machines”, Elsevier, (1984)

[2] W. Leonhard: “Control of Electrical Drives”, Springer-Verlag (1985)

[3] Paul C. Krause: “Analysis of Electric Machinery”, McGraw-Hill Book Company(1986)

[4] Peter Vas : "Electrical Machines and Drives : A Space-Vector Theory Approach”, Oxford Science Publications (1992)

[5] D.W.Novotny and T.A. Lipo: "Vector Control and Dynamics of AC Drives”, Oxford University Press (1996)

[6] S. Shinnaka: “ New "Mirror-Phase Vector Control” for Sensorless Drive of Permanent-Magnet Synchronous Motor With Pole Saliency”, IEEE Trans. Industr. Applic., Vol.40, No.2, pp.599-606 (2004) 